\title{
Impacts des grands herbivores sur la végétation des prairies et conséquences sur la décomposition de la litière
}

\author{
T. LEFEBVRE ${ }^{1,2}$, C. GALLET $T^{l}$ \\ 'Université Savoie Mont Blanc, CNRS, LECA, Laboratoire d'Écologie Alpine, 73000, Chambéry, France \\ ${ }^{2}$ Université Grenoble Alpes, CNRS, LECA, Laboratoire d'Écologie Alpine, 38000 Grenoble, France
}

Courriel : tiphaine.lefebvre.uds@gmail.com

Les herbivores influencent la végétation prairiale par le biais de la défoliation, du dépôt d'excréments et du piétinement. Face à ces perturbations, les plantes ont mis en place des stratégies de défense pouvant altérer leurs caractéristiques physico-chimiques. L'herbivorie influence également la structure de la communauté végétale. Ces effets peuvent modifier le fonctionnement de l'écosystème via la décomposition des litières. ${ }^{1}$

Les herbivores, définis par leur capacité à tirer leur énergie de la consommation de plantes, façonnent les communautés végétales dans lesquelles ils évoluent et dont ils se nourrissent. À large échelle, ils peuvent par exemple modifier la composition floristique ou encore la productivité des communautés végétales (Olofsson et Oksanen 2002). À plus fine échelle, ils peuvent influencer le métabolisme des plantes en induisant notamment la production de défenses, dont l'impact sur la qualité des plantes en tant que ressources fourragères sont souvent délétères (Kaplan et al 2008, Mithöfer et Boland 2012). Par effet de cascade, ces modifications peuvent à leur tour affecter la décomposition des litières et les cycles biogéochimiques qui en découlent, avec des conséquences pour les services agronomiques, tels que la qualité des fourrages ou la fertilité du sol (Bardgett et Wardle 2003).

Parmi les nombreuses études traitant d'interactions entre plantes et herbivores, un grand nombre d'entre elles concernent les herbivores invertébrés, et plus particulièrement les insectes phytophages. En effet, ils représentent une source d'herbivorie importante, de par leur grande diversité et leurs forts effectifs, et engendrent souvent des pertes massives de production dans les systèmes de cultures. Leur petite taille et leur cycle de reproduction rapide en font également des modèles d'étude faciles à manipuler lors d'expérimentations contrôlées ou semi- contrôlées. Contrairement aux herbivores invertébrés, les grands mammifères herbivores (i.e. dont la masse est supérieure à $2 \mathrm{~kg}$; Fritz et Loison 2006) prélèvent chez les plantes des feuilles entières, voire des individus entiers, en un laps de temps plus restreint (Hester et al 2006). Les effets macroscopiques des herbivores sur la végétation, incluant des modifications de la diversité, de la productivité ou encore du développement phénologique des communautés végétales, ont été largement étudiés. À l'inverse, les connaissances actuelles des mécanismes de défense des plantes induits par l'herbivorie, via l'investigation de la composition chimique des plantes, proviennent en grande majorité de la littérature concernant les herbivores invertébrés (Hester et al 2006).

L'objectif de ce présent article est de faire la synthèse de la littérature traitant de l'impact des grands herbivores sauvages et domestiques sur les plantes. La première partie de cette revue détaillera les mécanismes par lesquels ces herbivores affectent les plantes. La deuxième partie sera consacrée aux principales stratégies mises en place par les plantes pour minimiser les conséquences négatives de l'herbivorie sur leur survie et reproduction. Enfin la troisième partie développera les mécanismes par lesquels les herbivores peuvent affecter le fonctionnement des écosystèmes, via la décomposition des litières. En effet, ce processus est essentiel puisqu'il alimente le cycle des nutriments et constitue l'une des principales sources d'énergie disponible pour la croissance des plantes et donc pour l'ensemble de la chaine trophique.

\section{1 / Comment les herbivores affectent-ils les plantes ?}

Lorsque l'on évoque les effets des herbivores sur la végétation, on pense souvent à tort aux seules conséquences de la défoliation. Pourtant, la présence d'herbivores se traduit par un ensemble d'actions : la défoliation, le piétinement, le dépôt d'excréments au sol ou encore de salive sur les parties aériennes (figure 1). Chacun de ces processus est susceptible d'engendrer des mécanismes de réponse chez les plantes, dont la nature et l'intensité peuvent être modulées selon l'intensité d'herbivorie et l'identité des herbivores, mais également en fonction de caractéristiques propres à la végétation (ex. espèces végétales, stade phénologique...).

\section{1 / Défoliation}

Le pâturage par les grands herbivores implique une perte de biomasse végétale aérienne liée à la défoliation. À l'échelle de la plante, les dommages créés sont plus ou moins importants selon le stade phénologique ou les organes consommés et peuvent occasionnellement mener à la mort de la plante (Hester et al 2006). À l'échelle de la communauté végétale, 
Figure 1. Schéma conceptuel résumant les processus par lesquels les grands herbivores peuvent affecter la quantité et la qualité des plantes, et les différents mécanismes d'action impliqués.

Le signe « + » ou « - » indique si l'effet d'un item vers un autre est positif ou négatif. Pour plus de clarté, seules les interactions principales et les effets majoritaires sont représentés.

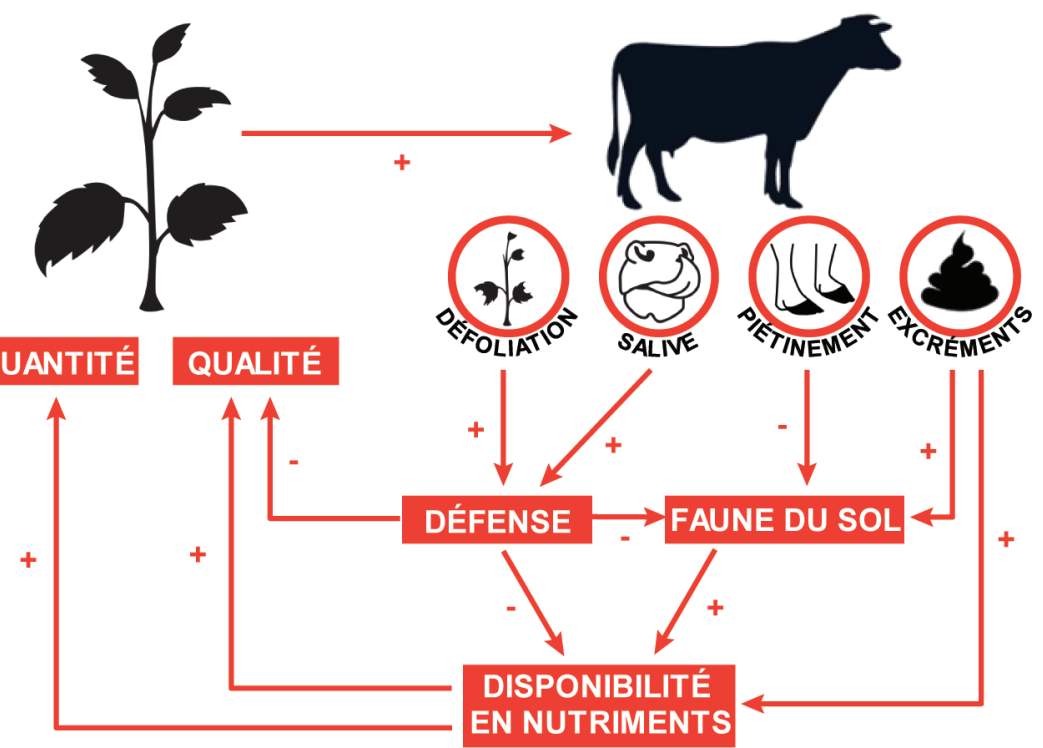

Encadré 1. Déterminants du régime alimentaire des grands herbivores.

Hofmann (1989) classe les grands herbivores ruminants en 3 types alimentaires, selon les caractéristiques morpho-physiologiques de leur rumen. À une extrémité de ce gradient se trouvent les « cueilleurs » (" browsers »; ex. chevreuil), dont le régime alimentaire est constitué essentiellement d'espèces dicotylédones ou d'organes de plantes sélectionnés pour leurs qualités nutritives. Leur rumen est peu adapté à la digestion des fibres mais la sécrétion d'enzymes par leurs glandes salivaires leur permet de faire face aux métabolites secondaires produits par les plantes (Hofmann et al 2008). À l'autre extrémité de ce gradient se trouvent les « paisseurs » ( grazers »; ex. vache), peu sélectifs et dont le régime alimentaire, constitué essentiellement de graminées, est riche en fibres. Ils compensent la faible qualité de leur alimentation en ingérant une plus grande quantité. Leur rumen, plus gros et plus musculeux que celui des " cueilleurs ", permet une digestion lente des fibres et une meilleure assimilation des nutriments. Entre ces deux extrêmes se trouvent les ruminants au régime intermédiaire (" intermediate feeders » ; ex. chamois).

Le régime alimentaire des grands herbivores dépend également de leur masse. En effet, le taux métabolique des herbivores est inversement proportionnel à leur taille (Demment et Van Soest 1985). Ainsi, les herbivores de petite taille ont besoin d'une alimentation de qualité pour satisfaire leurs besoins énergétiques et sélectionnent les espèces végétales riches en énergie et facilement digestibles. À l'inverse, les herbivores les plus grands consomment les espèces végétales en tenant peu compte de leurs caractéristiques physico-chimiques (pas ou peu de sélection).

toutes les espèces ne subissent pas la même intensité de défoliation par les herbivores : certaines sont consommées, à des degrés variables, alors que d'autres sont au contraire évitées. En effet, les herbivores sélectionnent les plantes selon leurs caractéristiques physiques ou chimiques, dans le but de couvrir leurs besoins nutritionnels (Demment et Van Soest 1985). Par exemple, les chamois en prairies subalpines préfèrent les plan- dépend de l'espèce d'herbivore considérée, les conséquences de la défoliation sur la végétation peuvent donc être contrastées selon les espèces d'herbivores. Ce processus de sélection peut, à long terme, être à l'origine d'un changement de composition floristique des communautés végétales, favorisant l'augmentation de l'abondance des plantes nonconsommées par les herbivores ou de celles tolérant l'herbivorie (Pastor et al 1993, Olofsson et Oksanen 2002).

Outre les blessures mécaniques liées à la défoliation, la salive déposée par les herbivores sur les plantes lors de leur consommation peut induire des changements biochimiques au sein des feuilles et contribuer à des modifications de la vitesse de croissance (Liu et al 2012). La quantité de salive produite varie selon les grands herbivores, en raison de la taille de leurs glandes salivaires (Hofmann et al 2008). La composition de la salive varie également selon les espèces. Par exemple, chez les grands herbivores ruminants, les protéines capables de complexer les tannins issus des végétaux sont présentes dans la salive des cerfs mulet (Odocoileus hemionus) et absentes dans celle des bovins (Bos taurus) et des moutons (Ovis aries) (Austin et al 1989). Plus généralement, ces protéines seraient présentes dans la salive des « cueilleurs » («browsers »), leur permettant de consommer des plantes riches en tannins, et absentes de la salive des « paisseurs » (« grazers ») (Shimada 2006). Cette spécificité des caractéristiques salivaires, variable d'une espèce de mammifère herbivore à une autre, peut être responsable de l'induction de défenses variées chez les plantes, comme c'est le cas chez les insectes phytophages dont les sécrétions orales contiennent de nombreux éliciteurs insectes-spécifiques (Kessler et Baldwin 2002).

\section{2 / Dépôt d'excréments}

Le dépôt d'excréments solides (fèces) par les herbivores peut directement affecter les plantes par des dommages physiques (Bardgett et Wardle 2003, Hester et al 2006), en particulier lorsqu'ils sont de grande taille et d'un poids conséquent. C'est le cas des déjections bovines et équines, qui limitent la croissance des plantes en étouffant la végétation et en bloquant l'accès à la lumière (Bloor et al 2012). Les fèces peuvent aussi présenter un caractère phytotoxique, via certains composés aromatiques (indole) ou azotés (ex. cyanide), inhibant par exemple le processus de germination ou limitant la croissance racinaire (Hoekstra et al 2002). De même, l'urine des herbivores peut créer des « brûlures » des racines végétales, causées par la toxicité de l'azote ammoniacal et l'importante concentration 
en sel de ce type d'excrément (Haynes et Williams 1993).

Cependant, les effets principaux des excréments seraient surtout indirects et positifs, grâce à l'accélération du cycle des nutriments, favorisant l'augmentation de la croissance des plantes et de la teneur en nutriments des tissus végétaux (Bloor et al 2012). En effet, les excréments sont riches en nutriments, principalement en azote et potassium, provenant essentiellement des excréments liquides. Par exemple, chez les vaches laitières, plus de $50 \%$ de l'azote ingéré est libéré via l'urine, et environ $25 \%$ via les fèces. De même, plus de $80 \%$ du potassium ingéré est libéré via l'urine, et $10 \%$ via les fèces (Haynes et Williams 1993). La disponibilité des nutriments pour les plantes dépend de la forme sous laquelle ils sont excrétés. L'azote des fèces des herbivores est principalement sous forme organique, issu de microorganismes ou d'aliments non-digérés. L'azote issu de l'urine est essentiellement présent sous forme d'urée, rapidement hydrolysable en ammonium puis nitrifiés en nitrates, deux formes directement assimilables par les plantes (Haynes et Williams 1993). Les excréments, et plus particulièrement les fèces, sont aussi riches en calcium, phosphore et magnésium (Haynes et Williams 1993), éléments indispensables à la croissance des plantes.

Cet enrichissement du sol entraîne d'une part l'augmentation de la disponibilité des nutriments directement assimilables par les plantes, et d'autre part contribue à stimuler l'activité de la biomasse microbienne favorable à la minéralisation de l'azote (Stark et al 2000). Les interactions entre espèces végétales peuvent ainsi s'en retrouver modifiées et provoquer des changements de la structure des communautés végétales (Bardgett et Wardle 2003). Le dépôt d'excréments par les grands herbivores peut aussi faciliter la dispersion des graines à des distances importantes de la plante dont elles sont issues (endozoochorie) et favoriser leur germination (Milotić et Hoffmann 2016).

Les excréments peuvent avoir des compositions et donc des conséquences variables selon les espèces d'herbivores, s'expliquant entre autres par la variabilité des régimes alimentaires et des caractéristiques des systèmes digestifs (Clauss et al 2002, Bakker et al 2004). Dans leur étude portant sur l'influence de trois espèces d'herbivores (bovins, lapins, campagnols des champs) sur le cycle de l'azote, Bakker et al (2004) ont montré que l'exclusion des bovins entraînait une augmentation de la minéralisation de l'azote sous l'action du pâturage par les deux autres herbivores. L'une des raisons évoquées par les auteurs concerne la spécificité des excréments des trois espèces. En effet, les excréments diffèrent par leur quantité, leur qualité, mais également par leur taille et la façon dont ils sont distribués. Ils émettent l'hypothèse que la distribution de l'azote par les petits herbivores serait plus homogène car leurs excréments sont de petite taille et répartis de manière plus uniforme. Par ailleurs, plus les excréments sont petits, plus leur décomposition est rapide et plus la disponibilité en azote pour les plantes est importante (Bakker et al 2004). D'autres travaux ont souligné l'importante hétérogénéité de distribution spatiale des excréments d'herbivores domestiques, fortement influencée par la gestion de la pâture et par le comportement des animaux. Les déjections des bovins et ovins sont souvent rassemblés autour de points d'attraction de la prairie, accumulant à ces endroits une quantité importante en nutriments (Bloor et al 2012).

\section{3 / Piétinement}

Le piétinement est l'un des mécanismes lié au pâturage par lequel les grands herbivores peuvent engendrer des modifications de la végétation (Xu et al 2013), du sol (Bilotta et al 2007) et des processus qui s'y déroulent (Schrama et al 2013). Il provoque la destruction de tissus végétaux, pouvant conduire à la mort d'une partie ou de la totalité de la plante (Hester et al 2006). Il crée alors des surfaces de sol nu, propice à la germination et à l'établissement de nouveaux individus (Faust et al 2011) et peut favoriser l'extension spatiale des individus par la stimulation de la production de parties végétatives (Sui et al 2011). Le piétinement peut aussi influencer les plantes de façon indirecte. Par exemple, la compaction du sol peut diminuer sa porosité et limiter la disponibilité en eau pour les plantes (Schrama et al 2013). Il peut également affecter l'activité des communautés biotiques impliquées dans les cycles géochimiques et ainsi modifier la disponibilité des nutriments utilisables par les plantes (Schrama et al 2013).

L'impact du piétinement sur la végétation varie selon les espèces de grands herbivores et augmente avec leur taille (Cumming et Cumming 2003). Contrairement à ce que l'on pourrait penser, les effets ne dépendent pas directement de la pression exercée au sol par les herbivores. La taille des herbivores est en fait proportionnelle à celle de leurs sabots, et la pression exercée au sol par unité d'aire est donc identique quelle que soit la taille des herbivores (Ssemakul 1983, Cumming et Cumming 2003). Par contre, la taille des herbivores est inversement proportionnelle à celle de leurs pattes. Ainsi, les herbivores de grande taille ont besoin de faire plus de pas que ceux de petite taille pour parcourir une même distance, relativement à leurs tailles, impliquant un piétinement plus important (Cumming et Cumming 2003). L'impact du piétinement sur le sol augmente également lorsque la densité en herbivores est importante (Bilotta et al 2007). Cela est particulièrement vrai dans le cas des herbivores domestiques ou semidomestiques, lorsque la gestion des troupeaux est inadaptée aux milieux qui les accueillent (Ssemakul 1983). Les comportements propres aux différentes espèces d'herbivores, tels que la tendance à se concentrer dans certaines zones préférées (reposoirs, abris...) ou encore les modalités de leurs déplacements (distance parcourue par jour, trajets empruntés...), participent aussi à expliquer la variabilité d'effets sur le sol des différentes espèces d'herbivores (Bilotta et al 2007, Nolte et al 2015).

\section{2 / Stratégies des plantes face au risque d'herbivorie}

En tant qu'organismes sessiles, les plantes n'ont pas la possibilité de fuir leurs prédateurs. Pour maximiser leurs chances de survivre et de se reproduire malgré la présence d'herbivores, les plantes ont développé trois stratégies principales : l'évitement, la tolérance et la résistance (figure 2).

\section{1 / Stratégie d'évitement}

La stratégie d'évitement est certainement la moins étudiée de toutes, et particulièrement concernant les grands herbivores puisque la majorité des preuves apportées par la littérature proviennent d'études sur les herbivores invertébrés. Cette stratégie consiste à réduire la probabilité d'une plante à être trouvée et donc consommée par les herbivores.

On distingue deux types d'évitement. L'évitement est dit « interne » dans le cas où les propres caractéristiques d'une plante lui permettent de se protéger des herbivores (Milchunas et Noy-Meir 2002). Il peut alors s'agir d'un évitement temporel se manifestant par des modifications phénologiques, rendant temporairement inaccessible les organes importants en terme de fitness (ex. fleurs) lorsque les prédateurs sont présents (Parachnowitsch et al 2012). Il peut aussi s'agir d'un évitement spatial, dans le cas où les traits morphologiques de la plante compliquent leur prélèvement par les herbivores (ex. plantes en rosette) ou si la plante pousse dans un endroit inaccessible. Par exemple, certaines plantes assurent leur protection contre les herbivores grâce à leur propriété grimpante, les mettant hors de portée des herbivores du sol, comme dans 
Figure 2. Schéma des trois stratégies de défense des plantes contre les herbivores et principaux mécanismes mis en œuvre.

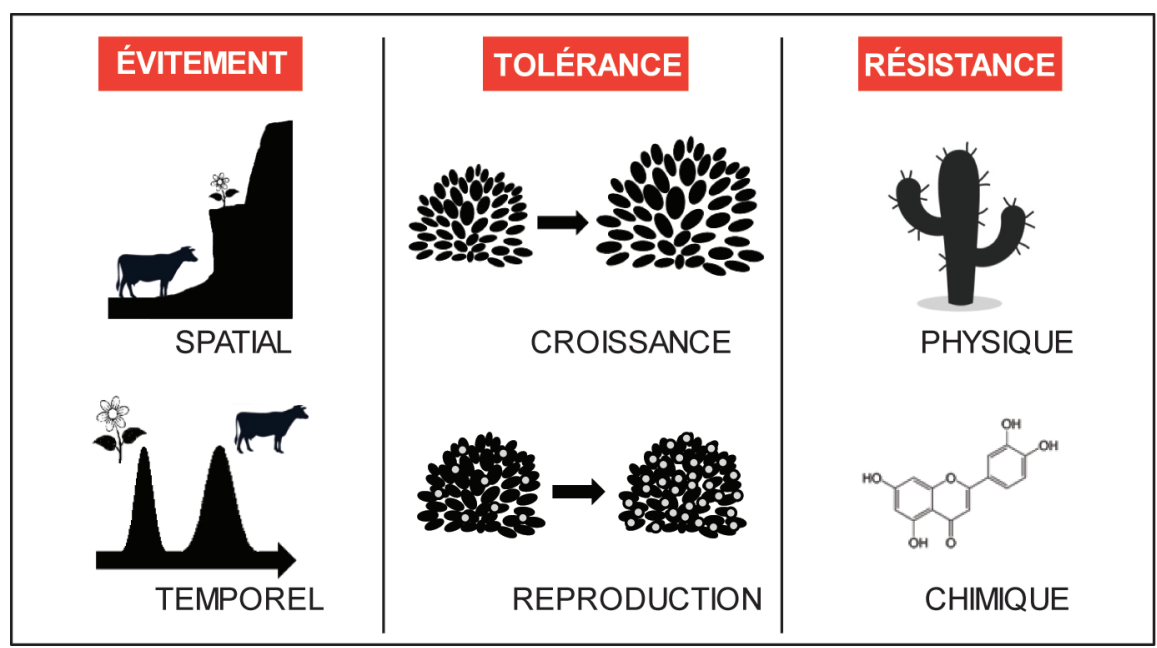

le cas de la vigne Boquila trifoliolata. Cette dernière voit aussi sa protection assurée par un phénomène de mimétisme puisque la forme, la taille et la couleur de ses propres feuilles " miment 》 les feuilles des arbres sur lesquels elle se trouve (Gianoli et Carrasco-Urra 2014).

L'évitement est dit « externe »s'il ne dépend pas des caractéristiques d'une plante, mais d'éléments biotiques ou abiotiques qui l'entourent (Milchunas et Noy-Meir 2002). Par exemple, une plante appréciée des herbivores peut éviter ses prédateurs grâce à ses plantes voisines. Lorsque l'herbivore sélectionne son alimentation à l'échelle du patch alimentaire, une plante peut bénéficier du voisinage d'espèces peu appréciées des herbivores, puisque le patch sera délaissé. À l'inverse, lorsque l'herbivore sélectionne son alimentation à l'échelle de l'élément fourrager, une plante pourra bénéficier de plantes voisines très appréciées des herbivores, puisque ces dernières seront consommées en priorité (Huang et al 2016). L'évitement externe peut aussi être assuré par la présence de refuges, offrant une protection physique aux plantes qui en bénéficient. Ces refuges peuvent être biotiques lorsqu'ils sont assurés par d'autres plantes, par exemple dans le cas de plantes poussant sous des buissons (Milchunas et Noy-Meir 2002). Ils peuvent aussi être géologiques, de taille plus ou moins grande, allant de petites anfractuosités d'un rocher inaccessibles à la bouche de grands herbivores, à des îles dépourvues d'herbivores, en passant par des falaises trop abruptes pour être foulées (Milchunas et Noy-Meir 2002).

\section{2 / Stratégie de tolérance}

La stratégie de tolérance permet aux plantes de maintenir leur fitness en favorisant la croissance et la reproduction pour pallier les dommages occasionnés par les herbivores (Strauss et Agrawal 1999). Par définition, cette stratégie devrait être mesurée soit par la pente de corrélation entre la fitness des plantes et le niveau de dommages constatés dans le cas où le niveau de dommage est continu, soit par la différence de fitness entre des individus de plantes endommagés et des individus non-endommagés dans le cas où le niveau de dommage expérimentalement engendré est fixe (Strauss et Agrawal 1999). Dans les faits, elle est plutôt évaluée par la mesure de certains mécanismes, développés cidessous, permettant à la plante de tolérer l'herbivorie.

Chez une plante ayant subit une défoliation partielle, l'augmentation de l'activité photosynthétique des tissus végétaux épargnés par l'attaque est l'un des mécanismes de tolérance (Strauss et Agrawal 1999, Thomson et al 2003). Il permet en effet d'accroître la production de carbohydrates, fournissant l'énergie nécessaire aux processus cellulaires de la plante qui pourraient permettre de compenser la perte de tissus engendrée par les herbivores. Les résultats des quelques études ayant exploré l'influence des grands herbivores sur l'activité photosynthétique des plantes sont contrastés, mettant en évidence une augmentation ou au contraire une diminution du contenu en chlorophylle des plantes, respectivement interprétés comme étant le reflet d'un mécanisme de compensation ou d'une altération de l'état physiologique (Redondo-Gómez et al 2010, Pellissier 2013).

La stratégie de tolérance peut aussi se manifester par le remplacement de tissus prélevés par les herbivores, grâce à l'activation des méristèmes. Cette croissance dite compensatoire peut par exemple se traduire par une élongation des tiges (Champagne et al 2012) ou encore du nombre de feuilles (Korpita et al 2014), et peut s'accompagner d'une modification d'allocation des ressources au sein de la plante. Par exemple, Newingham et al (2007) ont observé un transfert des ressources azotées des racines vers les feuilles chez des individus de Centaurea maculosa exposés à des herbivores racinaires.

L'augmentation des capacités reproductrices des plantes endommagées par les herbivores est aussi caractéristique de la stratégie de tolérance. Dans leur étude, Martin et al (2014) ont montré que les individus d'Impatiente du Cap (Impatiens capensis) pâturés par les cerfs de Virginie (Odocoileus virginianus) étaient plus tolérants que les individus protégés de ces herbivores, notamment grâce à une augmentation du nombre de fruits par individus. La modification du développement phénologique des plantes représente aussi un mécanisme par lequel les plantes peuvent tolérer l'herbivorie. Par exemple, les individus d'Impatiente du Cap pâturés par les cerfs de Virginie ont un nombre de jours de floraison supérieur à celui des individus protégés des herbivores (Martin et al 2014).

\section{3 / Stratégie de résistance}

La stratégie de résistance permet aux plantes de limiter la probabilité d'être consommées par les herbivores, grâce à l'expression de traits qui réduisent la préférence ou la performance des herbivores (Strauss et Agrawal 1999). Ces traits de résistance ont été classés de multiples façons par les auteurs : en fonction de leur structure (physique ou chimique), de leur mode de production (résistance constitutive ou induite), ou encore leur mode d'action (direct ou indirect).

Les composés de résistance physique dissuadent les herbivores de consommer les plantes qui en sont pourvues (Hanley et al 2007). Certains sont macroscopiques, tels que les épines ou les trichomes, d'autres sont microscopiques, comme la silice. Cette dernière est contenue dans les parois cellulaires des végétaux ou sous forme d'agrégats abrasifs (phytolithes), essentiellement dans les feuilles de monocotylédones, avec une concentration pouvant atteindre jusqu'à $5 \%$ de leur masse sèche, soit 10 à 20 fois la concentration en silice des feuilles d'espèces dicotylédones (Massey et al 2006). La résistance chimique est essentiellement assurée par les nombreux composés organiques appelés métabolites secondaires assurant entre autres un rôle répulsif ou toxiques vis-à-vis des herbivores. On distingue plusieurs dizaines de milliers de molécules différentes, classiquement regroupées en trois grandes classes : les terpènes, les composés azotés comprenant les alcaloïdes et les composés phénoliques (encadré 2). 
Encadré 2. Les métabolites secondaires des plantes.

Les terpènes constituent la plus grande classe de composés secondaires (plus de 30000 composés identifiés) et sont ubiquistes au sein du règne végétal (Mithöfer et Boland 2012). Les rôles des terpènes dans les plantes sont variés et dépendent de leur degré de polymérisation. Les plus petits d'entre eux participent aux odeurs émises par les végétaux (ex. $\alpha$ - et $\beta$-pinène chez les végétaux de la famille des Pinaceae), certains constituent les précurseurs d'hormones végétales indispensables au développement des plantes, d'autres jouent un rôle dans la défense contre les herbivores (exemple des latex) ou au contraire ont un pouvoir attractif sur certains insectes (Junker et Blüthgen 2010).

Les composés phénoliques sont ubiquistes dans le règne végétal, avec plus de 9000 composés dénombrés (Mithöfer et Boland 2012). Ils assurent des rôles très diversifiés, d'une part à l'échelle cellulaire en protégeant par exemple les feuilles contre les UV, et d'autre part à une échelle beaucoup plus large, permettant à la plante d'interagir avec son milieu, en favorisant par exemple l'attraction des pollinisateurs ou encore en agissant comme composés de défense contre les herbivores (Hättenschwiler et Vitousek 2000). Parmi les composés phénoliques ayant une activité anti-herbivorie, les tannins, polymères hydrosolubles, sont certainement les plus connus. Ils réduisent la digestibilité du fourrage par les herbivores en formant des complexes insolubles avec les protéines des plantes ou celles des enzymes du système digestif (Mueller-Harvey 2006).

Les composés azotés sont essentiellement représentés par les alcaloïdes (plus de 12000 composés répertoriés). Ils se retrouvent dans environ $20 \%$ des espèces de plantes vasculaires (très représentés chez les Solanaceae) et chez quelques champignons (Mithöfer et Boland 2012). Les alcaloïdes assurent principalement un rôle de défense contre les prédateurs, grâce à leur caractère toxique et répulsif, même en très faible quantité (Pfister et al 2001). D'autres composés azotés tels que les glucosinolates, les composés cyanogénétiques ou encore les inhibiteurs de protéases (Mithöfer et Boland 2012) jouent aussi un rôle de défense. Ces derniers sont des polypeptides ayant la capacité de limiter le processus de digestion grâce au blocage des activités enzymatiques (Lindgren et al 2007).

Ces composés de résistance (qu'ils soient physiques ou chimiques) peuvent être constitutifs ou induits, selon leur mode de production. Les composés constitutifs sont définis par Agrawal (2007) comme étant des traits de plantes qui sont toujours exprimés, même en absence d'herbivores, alors que les composés induits sont des traits dont la production ou l'activité anti-herbivore est augmentée à la suite d'une attaque dans le but de réduire la probabilité d'une attaque ultérieure. L'induction de certains composés peut être très rapide et mesurable après quelques jours (voire heures) après que la plante ait été blessée, comme l'ont montré plusieurs études simulant l'herbivorie (Lindgren et al 2007, Kaplan et al 2008). La frontière entre les deux types de composés est loin d'être stricte, puisque la concentration de nombreux composés constitutifs peut augmenter suite à l'attaque d'herbivores (Kaplan et al 2008).

Les composés de résistance ont généralement un effet délétère sur les herbivores par des modes d'actions directs ou indirects. Les composés à activité « directe » peuvent agir en tant que répulsif, en altérant la texture (ex. épines), le goût ou l'odeur (ex. terpènes) de la plante. D'autres comme la lignine ou les tannins vont limiter le processus de digestion, et réduire la valeur nutritive des plantes en agissant comme barrière à l'action des enzymes de dégradation, respectivement des polysaccharides et des protéines (Moore et Jung 2001). Enfin, une grande majorité d'entre eux (alcaloïdes notamment) induisent une toxicité plus ou moins prononcée chez les animaux (Provenza et al 2007). Certains composés dits de résistance peuvent néanmoins être bénéfiques pour l'animal s'ils sont consommés en petite quantité. Par exemple, les tannins condensés ont une action anthelminthique et permettent une meilleure assimilation des acides aminés par les ruminants, en favorisant la digestion des protéines dans le petit intestin plutôt que dans le rumen (Mueller-Harvey 2006). De plus, les résistances chimiques induites ne sont efficaces que si les herbivores parviennent à les détecter et à être suffisamment sélectifs pour éviter de consommer ces plantes. Les composés à activité « indirecte» vont protéger la plante de ses agresseurs herbivores en faisant appel leurs prédateurs (Turlings et al 1990). Ce processus est maintenant bien connu chez les insectes phytophages mais a aussi été identifié dans le cas de mammifères herbivores. Par exemple, des acacias exposés aux grands herbivores de savane africaine induisent la produc- tion de nectar, responsable de l'attraction de fourmis dont l'agressivité entraine une diminution de l'herbivorie (Huntzinger et al 2004).

\section{3 / Effet des herbivores sur la décomposition des litières}

Dans les écosystèmes terrestres, la majorité de la production primaire retourne au sol sous forme de litière (jusqu'à 90\%; Chapin et al 2011). Ce processus est essentiel au fonctionnement des écosystèmes puisqu'il alimente le cycle des nutriments et impacte ainsi les services écosystémiques associés tels que la productivité primaire ou le stockage du carbone. Trois facteurs principaux contrôlent le processus de décomposition : la qualité des litières, les communautés d'organismes décomposeurs et le climat (Chapin et al 2011). Dans les sous-parties suivantes, nous verrons que les herbivores ont la capacité d'influencer le processus de décomposition, via des modifications de la qualité des litières, des communautés de décomposeurs, ou des conditions microclimatiques du sol (figure 3).

\section{1 / Effet des herbivores par altération de la qualité des litières}

La qualité d'une litière, définie par sa composition biochimique, détermine en grande partie son taux de décomposition. De façon simplifiée, une litière contenant des molécules de faibles poids moléculaire facilement dégradables (ex. carbohydrates) sera facilement décomposable et sera donc dite de bonne qualité. À l'inverse, une litière riche en polymères peu dégradables (ex. lignine) sera récalcitrante à la décomposition et sera donc dite de mauvaise qualité.

La qualité des litières est une conséquence directe de la composition biochimique des plantes dont elles sont issues (Kurokawa et Nakashizuka 2008), particulièrement dans le cas où le lessivage et la résorption des nutriments par la plante lors de la senescence des feuilles sont négligeables (Chapin et al 2011). En tant que consommateurs primaires, les herbivores peuvent modifier la qualité des litières en altérant la composition chimique des plantes soit via la modification de traits à court terme, soit en changeant les proportions relatives des différents tissus de plantes, ou enfin en changeant l'abondance relative des espèces dans la communauté végétale, voire l'assemblage des espèces (figure 3 ). Ces trois processus peuvent, par effet de cascade, impacter la qualité de la litière et donc sa décomposition. On parle alors d'effets d'héritage ou post-mortem (« legacy effects » ou « afterlife effects »), 
Figure 3. Schéma conceptuel des trois facteurs principaux (encadrés rouges) contrôlant le processus de décomposition des litières (symbolisé par la double flèche) et des moyens par lesquels les herbivores peuvent influencer chacun de ces facteurs (encadrés gris).

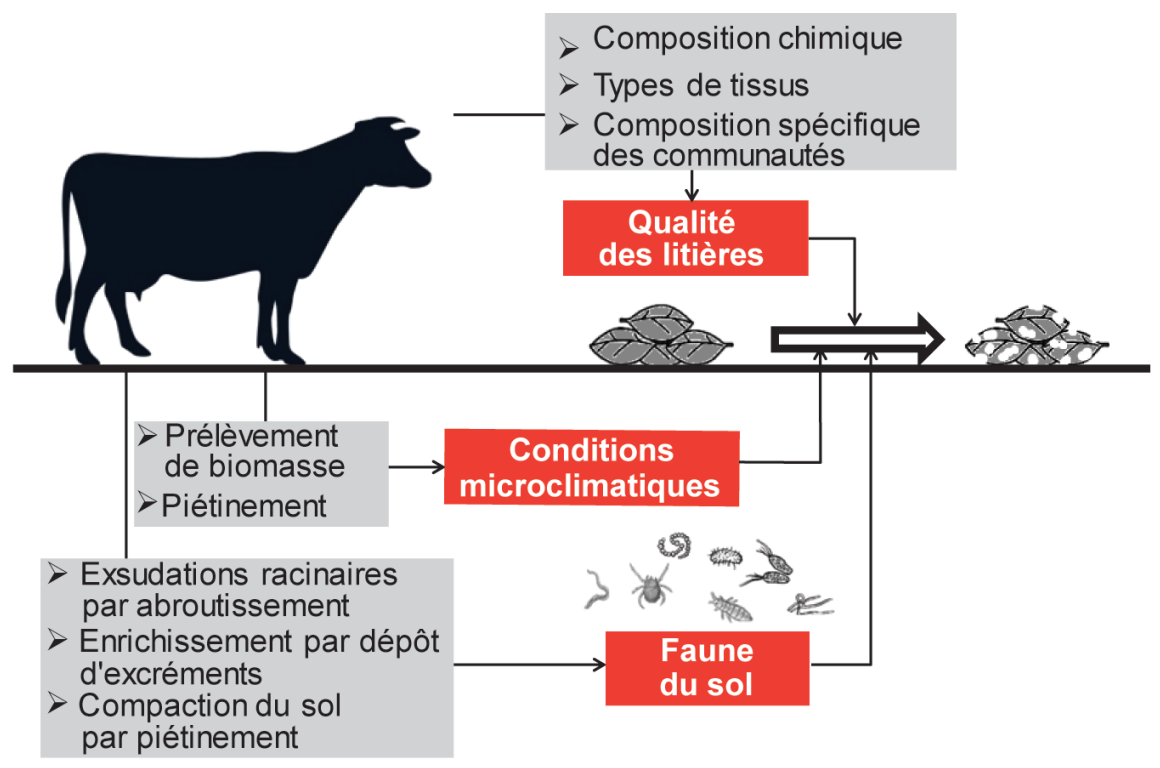

définis par Han et al (2014) comme étant les conséquences de perturbations antérieures qui continuent d'affecter la structure et la fonction d'un écosystème.

Un changement des traits physiques, chimiques ou encore morphologiques des plantes par les herbivores peut modifier la qualité des litières et impacter en retour le processus de décomposition. Ce processus s'expliquerait par l'existence de traits végétaux qui sont à la fois susceptibles d'être induits par les herbivores, et également impliqués dans le contrôle du processus de décomposition (Wardle et al 2002). Par exemple, les composés phénoliques peuvent être induits par les herbivores à des fins de défense, mais sont aussi connus pour contrôler la vitesse de décomposition de certaines litières. Certains auteurs ont mis en évidence que ces composés pouvaient être responsables d'une baisse (Hättenschwiler et Vitousek 2000) ou d'une augmentation du taux de décomposition des litières (Ibanez et al 2013), alors que d'autres n'ont pas constaté d'effet (Kurokawa et Nakashizuka 2008). Cette diversité de réponses s'expliquerait par la grande diversité des composés phénoliques et de leurs caractéristiques physico-chimiques. Certains composés de faibles poids moléculaires (tels que les acides phénoliques ou certains flavonoïdes) et hydrosolubles stimuleraient la décomposition des litières en servant de ressources carbonées aux activités microbiennes (Schimel et al 1998). Au contraire, la présence de polymères de plus haut poids moléculaire (tels que les tannins ou la lignine) ralentirait la décomposition des litières, par la formation de complexes insolubles et récalcitrants à la décomposition par la plupart des orga- nismes décomposeurs (Hättenschwiler et Vitousek 2000).

En sélectionnant certaines parties de plantes, les herbivores peuvent modifier les proportions relatives des différents organes qui retourneront au sol lors de la sénescence. Dans leur méta-analyse portant sur la décomposabilité de litières à travers le monde, Freschet et al (2013) ont estimé qu'en milieu prairial, $26 \%$ de la litière produite proviendrait des feuilles, $41 \%$ des tiges et $33 \%$ des racines. Par ailleurs, selon cette même étude, la décomposabilité des feuilles est 1,5 fois supérieure à celle des tiges, et 2,8 fois supérieure à celle des racines. Ainsi, la sélection à fine échelle d'organes de plantes par les herbivores peut changer la proportion de ces organes dans la litière, et ainsi influencer le processus de décomposition.

Sur le long terme, la sélection alimentaire des herbivores peut influencer la structure des communautés végétales et favoriser l'expression de traits foliaires associés à des taux de décomposition réduits (Wardle et al 2004). Par exemple, Pastor et al (1993) ont montré que le pâturage par les élans (Alces alces) en milieu boréal était à l'origine d'un remplacement progressif d'espèces végétales $P$. mariana), peu appréciés des herbivores et produisant une litière de faible qualité, cette dernière étant elle-même à l'origine d'une diminution de la qualité du sol. L'impact des herbivores sur la décomposition des litières via un changement de composition floristique pourrait s'expliquer par l'existence de traits végétaux influençant à la fois le processus de décomposition mais aussi le palatables par des épicéas (Picea glauca, choix alimentaire des herbivores. Par exemple, Bison (2015) a montré que les chamois sélectionnaient les plantes caractérisées par une forte teneur en azote et une faible teneur en matière sèche. Or les plantes riches en azote (Cornwell et al 2008) et celles à faible teneur en matière sèche (Bumb 2016) ont une décomposabilité supérieure aux plantes caractérisées par des valeurs de traits opposées.

Les effets du pâturage sur la décomposition des litières, via une modification de l'abondance relative des espèces dans la communauté végétale, peuvent être amplifiés dans le cas où cette modification s'accompagne d'un changement de groupes fonctionnels (Semmartin et al 2004). En effet, la décomposabilité des litières varie fortement selon ces groupes. Dans un ordre décroissant de décomposabilité des litières, on trouve généralement : les herbacées non-graminoïdes, les herbacées graminoïdes, les laîches, les buissons décidus, les buissons sempervirents et les mousses (Cornelissen et al 1999, Cornwell et al 2008). Les études taxonomiques confortent ces observations. À l'échelle globale, les bryophytes et ptéridophytes sont généralement les taxons pour lesquels la décomposition est la plus lente, alors que les dicotylédones sont les taxons pour lesquels la décomposition des litières est la plus rapide. Les monocotylédones et gymnospermes ont quant à eux des valeurs intermédiaires de décomposition (Cornwell et al 2008). À une échelle spatiale plus réduite, des études s'intéressant uniquement à des espèces herbacées méditerranéennes ont montré que la décomposition des litières était minimale pour les Poaceae, intermédiaire pour les Lamiaceae et maximale pour les Asteraceae et Fabaceae (Birouste et al 2012). Il est à noter que ces deux dernières familles dont la décomposabilité est importante sont par ailleurs très prisées des grands herbivores (Bison 2015).

\section{2 / Influence des herbivores sur les communautés biotiques du sol}

En tant que consommateurs de matière organique en sénescence, les organismes décomposeurs jouent un rôle essentiel dans le processus de décomposition des litières (Chapin et al 2011). La diversité des organismes participant au processus de décomposition est importante. De façon simplifiée, on distingue deux types d'acteurs : les microorganismes décomposeurs et les détritivores (Gessner et al 2010). Les microorganismes décomposeurs, acteurs principaux du processus de décomposition, sont composés de bactéries et champignons, permettant la transformation des structures polymérisées (ex. lignine) en molécules simplifiées (Berg et McClaugherty 2014). Les détritivores, rassemblant des orga- 
nismes tels que les collemboles, les lombrics ou encore les acariens, se nourrissent de débris de litière, assurant ainsi sa fragmentation, et/ou de bactéries et champignons.

De nombreuses études montrent que les herbivores (foliaires et racinaires) peuvent influencer les communautés biotiques du sol et ainsi impacter la décomposition de la litière (Sankaran et Augustine 2004, Bressette et al 2012). Les mécanismes impliqués sont nombreux et complexes car interconnectés les uns aux autres (Bardgett et Wardle 2003). De façon synthétique, les grands herbivores peuvent influencer les communautés biotiques du sol (et in fine le processus de décomposition) en modifiant des conditions abiotiques du sol et la disponibilité de ressources, via le piétinement, le dépôt d'excréments, la modification de la qualité des litières et l'induction d'exsudats racinaires.

Le piétinement impacte la faune du sol de façon directe, par l'écrasement d'individus à la surface du sol, mais également par plusieurs processus indirects, développés dans la revue de Bilotta et al 2007, impliquant la modification des conditions de vie des organismes du sol. Tout d'abord, l'altération des conditions hydriques et d'oxygénation du sol, sous l'effet de la réduction de la porosité, influence l'activité des organismes décomposeurs, en limitant par exemple les déplacements des détritivores tels que les lombrics (Bilotta et al 2007). Le piétinement de la végétation peut aussi altérer la quantité et la qualité des ressources alimentaires disponibles pour les organismes de sol. Enfin, la réduction du couvert végétal peut exposer d'avantage les organismes décomposeurs à leurs prédateurs. Les conséquences du piétinement des grands herbivores sur les caractéristiques du sol et l'abondance des organismes décomposeurs restent néanmoins difficilement généralisables, puisqu'il a notamment été montré qu'une même espèce d'herbivore peut avoir des effets variables en fonction du type de sol (Schrama et al 2013).

L'augmentation de la disponibilité des nutriments via le dépôt d'excréments peut localement augmenter la biomasse et l'activité des communautés microbiennes du sol (Stark et al 2000), stimulant localement et à court terme la décomposition des litières (Olofsson et Oksanen 2002). Ce processus est souvent caractérisé de " cycle court » des nutriments, et s'oppose au «cycle lent», par lequel le retour des nutriments au sol se fait via la sénescence des plantes et la décomposition des litières (Bardgett et al 1998, Bakker et al 2004). Les excréments déposés au sol par les herbivores domestiques d'élevage soumis à certains traitements anti- parasitaires peuvent aussi avoir d'importantes conséquences néfastes pour l'entomofaune coprophage (Bloor et al 2012).

Les caractéristiques de la faune du sol dépendent étroitement de la qualité des litières. Concernant les microorganismes, les litières dites de « bonne » qualité, issues de plantes caractéristiques de milieux productifs, seront typiquement associées à des communautés microbiennes dominées par les bactéries. À l'inverse, des plantes caractéristiques de milieux de faible productivité, produisant des litières récalcitrantes à la décomposition, seront associées à des communautés microbiennes dominées par les champignons (Bardgett et al 1998, Wardle et al 2004). En effet, les besoins en nutriments et l'activité métabolique des champignons sont moindres que ceux des bactéries (Hodge et al 2000). Ainsi, l'influence des herbivores sur la strate herbacée, à l'échelle de la plante par une modification des valeurs de traits, ou à l'échelle de la communauté végétale par un changement de sa composition spécifique, peuvent influencer les communautés d'organismes décomposeurs (Bardgett et Wardle 2003, Bressette et al 2012) et avoir des répercussions sur la décomposition des litières et le cycle des nutriments (Sankaran et Augustine 2004). La récente revue de Chomel et al (2016) insiste sur le rôle clé des métabolites secondaires dans le processus de décomposition, notamment par leurs effets directs sur les microorganismes et la faune du sol. Par exemple, la suppression de composés secondaires carbonés chez des lichens a eu pour conséquence une augmentation de l'abondance des acariens, des collemboles et des araignées (Asplund et al 2015). La toxicité ou encore la présence de structures résistantes à la biodégradation (ex. cycles aromatiques) seraient les raisons principales d'une limitation de la croissance et de l'activité des organismes détritivores (Chomel et al 2016).

Selon Bardgett et Wardle (2003), les substances carbonées issues de la photosynthèse allouées aux parties souterraines peuvent être stockées, utilisées pour la croissance ou la respiration racinaire, ou libérées dans la rhizosphère sous forme d'exsudats racinaires. La production de ces exsudats racinaires varie selon les espèces végétales, l'état physiologique de la plante et les conditions environnementales. Plusieurs études ont montré que l'exsudation racinaire pouvait aussi être stimulée par la défoliation, induite par les herbivores invertébrés (Holland et al 1996) et simulée de façon expérimentale par coupe manuelle des tissus végétaux (Hamilton et al 2008). Ces exsudats racinaires représentent une ressource facilement biodisponible et de haute qualité pour le développement des micro- organismes du sol (Bardgett et al 1998). En stimulant l'activité des communautés microbiennes du sol, ils favorisent ainsi le processus de décomposition des litières (Bardgett et Wardle 2003).

\section{3 / Impact des herbivores sur les conditions microclimatiques du sol}

Le climat est traditionnellement considéré comme étant un facteur important contrôlant la décomposition des litières, cette dernière étant généralement favorisée en conditions de température et de précipitations importantes (Chapin et al 2011). Les herbivores peuvent localement modifier les conditions microclimatiques du sol (Bardgett et Wardle 2003). Par exemple, le prélèvement de biomasse aérienne par les herbivores peut entraîner une augmentation de la température et une diminution de l'humidité du sol. En effet, la diminution de la couverture végétale est favorable à un réchauffement solaire du sol, qui a pour conséquence de favoriser l'évapotranspiration (Risch et al 2013). Ce changement de conditions microclimatiques est souvent invoqué comme étant une cause probable de l'augmentation de l'activité des communautés microbiennes et de l'accélération du processus de décomposition des litières en présence d'herbivores (ex. Stark et al 2000, Haynes et al 2014). La compaction du sol par le piétinement modifie également les conditions hydrologiques du sol en réduisant la teneur en eau, avec des conséquences potentielles négatives sur le cycle des nutriments (Bilotta et al 2007).

\section{Conclusion}

Les mécanismes par lesquels les grands herbivores peuvent influencer les communautés végétales dans lesquelles ils évoluent sont multiples, variables dans l'espace et dans le temps, et souvent interconnectés. Que ce soit par le biais de la défoliation, du piétinement ou des restitutions animales, les herbivores peuvent directement modifier la morphologie et le métabolisme des plantes et ainsi altérer la production de biomasse et la qualité du fourrage disponible. Ces dernières peuvent aussi être influencées indirectement par les herbivores via des rétroactions entre le sol et les plantes. En effet, la présence d'herbivores peut impacter les flux de matières et le processus de décomposition des litières, modifiant la disponibilité des nutriments pour les plantes.

Jusqu'à présent, les études traitant de l'impact des grands herbivores sur les plantes se sont essentiellement concentrées sur les caractéristiques macroscopiques de la végétation, tels que la diversité, la biomasse ou encore la phénologie. 
Les effets sur le métabolisme des plantes, et notamment sur les moyens de défense mis en place suite à l'attaque par les grands herbivores, sont plus rares puisque l'essentiel de la littérature, dans ce domaine précis, porte sur l'influence d'insectes phytophages ou de l'herbivorie simulée par arrachage ou coupe manuelle. De même, l'impact des composés de défense induits par les grands herbivores sur le processus de décomposition des litières et les cycles géochimiques qui s'ensuivent n'a été éva- lué que pour de rares espèces et dans un nombre très restreint d'écosystèmes. L'ensemble de ces connaissances pourrait permettre de mieux envisager les conséquences de changements de pratiques pastorales des herbivores domestiques et/ou des fluctuations de populations d'herbivores sauvages sur le fonctionnement des écosystèmes. De même, ces connaissances pourraient à terme éclairer certaines décisions de gestion afin de maintenir un équilibre durable entre populations d'herbivores et communautés végétales.

\section{Remerciements}

Les auteurs remercient Anne Farruggia de nous avoir encouragées à publier cette revue de la littérature ainsi que Grégory Loucougaray, René Baumont et les deux lecteurs anonymes pour leurs commentaires ayant permis d'améliorer la qualité de cette revue bibliographique.

\section{Références}

Agrawal A.A., 2007. Macroevolution of plant defense strategies. Trends Ecol. Evol., 22, 103 109.

Asplund J., Bokhorst S., Kardol P., Wardle D.A., 2015. Removal of secondary compounds increases invertebrate abundance in lichens. Fungal Ecol., 18, 18-25.

Austin P.J., Suchar L.A., Robbins C.T., Hagerman A.E., 1989. Tannin-binding proteins in saliva of deer and their absence in saliva of sheep and cattle. J. Chem. Ecol., 15, 1335-1347.

Bakker E.S., Olff H., Boekhoff M., Gleichman J.M., Berendse F., 2004. Impact of herbivores on nitrogen cycling: contrasting effects of small and large species. Oecologia, 138, 91-101.

Bardgett R.D., Wardle D.A., 2003. Herbivoremediated linkages between aboveground and belowground communities. Ecology, 84, 22582268.

Bardgett R.D., Wardle D.A., Wardle G., 1998. Linking above-ground and below-ground interactions: how plant responses to foliar herbivory influence soil organisms. Soil Biol. Biochem., 30, 1867-1878.

Berg B., McClaugherty C., 2014. Plant litter: decomposition, humus formation, carbon sequestration. $3^{\text {rd }}$ Ed. Springer-Verlag, Berlin, Germany, 315 p.

Bilotta G.S., Brazier R.E., Haygarth P.M., 2007. The impacts of grazing animals on the quality of soils, vegetation, and surface waters in intensively manages grasslands. Adv. Agron., 94, 237-280

Birouste M., Kazakou E., Blanchard A., Roumet C., 2012. Plant traits and decomposition: are the relationships for roots comparable to those for leaves? Ann. Bot., 109, 463-472.

Bison M., 2015. Approches taxonomique et fonctionnelle des interactions trophiques entre grands herbivores et communautés végétales dans un écosystème de montagne. $\mathrm{PhD}$ thesis, Université Grenoble Alpes \& Université de Trømso.

Bloor J.M.G., Jay-Robert P., Le Morvan A., Fleurance G., 2012. Déjections des herbivores domestiques au pâturage : caractéristiques et rôles dans le fonctionnement des prairies. INRA Prod. Anim., 25, 45-56.

Bressette J.W., Beck H., Beauchamp V.B., 2012. Beyond the browse line: complex cascade effects mediated by white-tailed deer. Oikos, 121, 1749-1760.

Bumb I., 2016. Impact des pratiques de gestion sur la qualité du fourrage dans les parcours méditerranéens du sud de la France : liens avec la décomposition de la litière. PhD thesis, Université de Montpellier, France.

Champagne E., Tremblay J.P., Côté S.D., 2012 Tolerance of an expanding subarctic shrub, Betula glandulosa, to simulated caribou browsing. Plos one, 7, e51940.

Chapin F.S., Matson P.A., Vitousek P., 2011 Principles of terrestrial ecosystem ecology. 2nd Edition. Springer, New York, USA, 529p.

Chomel M., Guittonny-Larchevêque M., Fernandez C., Gallet C., DesRochers A., Paré D. Jackson B.G., Baldy V., 2016. Plant secondary metabolites: a key trait for litter decomposition and soil nutrient cycling. J. Ecol., 102, 411-424.

Clauss M., Lechner-Doll M., Streich J.W., 2002. Faecal particle size distribution in captive wild ruminants: an approach to the browser/ grazer dichotomy from the other end. Oecologia, 131, 343-349.

Cornelissen J.H.C., Pérez-Harguindeguy N., Díaz S., Grime J.P., Marzano B., Cabido M., Vendramini F., Cerabolini B., 1999. Leaf structure and defence control litter decomposition rate across species and life forms in regional floras on two continents. New Phytol., 143, 191-200.

Cornwell W.K., Cornelissen J.H.C., Amatangelo K., Dorrepaal E., Eviner V.T., Godoy O., Hobbie S.E., Hoorens B., Kurokawa H., Pérez-Harguindeguy N., Quested H.M., Santiago L.S., Wardle D.A., Wright I.J., Aerts R., Allison S.D., van Bodegom P., Brovkin V., Chatain A., Callaghan T.V., Díaz S., Garnier E., Gurvich D.E., Kazakou E., Klein J.A., Read J., Reich P.B., Soudzilovskaia N.A., Vaieretti M.V., Westoby M., 2008. Plant species traits are the predominant control on litter decomposition rates within biomes worldwide. Ecol. Lett., 11, 1065-1071.

Cumming D.H.M., Cumming G.S., 2003 Ungulate community structure and ecological processes: body size, hoof area and trampling in African savannas. Oecologia, 134, 560-568.

Demment M.W., Van Soest P.J., 1985. A nutritional explanation for body-size patterns of ruminant and nonruminant herbivores. Am. Nat., 125, 641-672.
Edouard N., Duncan P., Dumont B., Baumont R., Fleurance G., 2010. Foraging in a heterogeneous environment - an experimental study of the trade-off between intake rate and diet quality. Appl. Anim. Behav. Sci., 126, 27-36.

Faust C., Eichberg C., Storm C., Schwabe A., 2011. Post-dispersal impact on seed fate by livestock trampling - a gap of knowledge. Basic Appl. Ecol., 12, 215-226.

Freschet G.T., Cornwell W.K., Wardle D.A., Elumeeva T.G., Liu W., Jackson B.G., Onipchenko V.G., Soudzilovskaia N.A., Tao J., Cornelissen J.H.C., 2013. Linking litter decomposition of above-and below-ground organs to plant-soil feedbacks worldwide. J. Ecol., 101, 943-952.

Fritz H., Loison A., 2006. Large herbivores across biomes. In: Large herbivore ecology, ecosystem dynamics and conservation. Danell K., Duncan P., Bergström R., Pastor J. (Eds). Cambridge University Press, New York, USA, 19-49.

Gessner M.O., Swan C.M., Dang C.K., McKie B.G., Bardgett R.D., Wall D.H., Hättenschwiler S., 2010. Diversity meets decomposition. Trends Ecol. Evol., 25, 372-380.

Gianoli E., Carrasco-Urra F., 2014. Leaf mimicry in a climbing plant protects against herbivory. Curr. Biol., 24, 984-987.

Hamilton E.W., Frank D.A., Hinchey P.M., Murray T.R., 2008. Defoliation induces root exudation and triggers positive rhizospheric feedbacks in a temperate grassland. Soil Biol. Biochem., 40, 2865-2873.

Han J., Chen J., Han G., Shao C., Sun H., Li L., 2014. Legacy effects from historical grazing enhanced carbon sequestration in a desert steppe. J. Arid Environ., 107, 1-9.

Hanley M.E., Lamont B.B., Fairbanks M.M., Rafferty C.M., 2007. Plant structural traits and their role in anti-herbivore defence. Perspect. Plant Ecol. Evol. Syst., 8, 157-178.

Hättenschwiler S., Vitousek P.M., 2000. The role of polyphenols in terrestrial ecosystem nutrient cycling. Trends Ecol. Evol., 15, 238-243.

Haynes R.J., Williams P.H., 1993. Nutrient cycling and soil fertility in the grazed pasture ecosystem. Adv. Agron., 49, 119-199.

Haynes A., Schütz M., Buchmann N., PageDumroese D., Busse M., Risch A., 2014. Link- 
ages between grazing history and herbivore exclusion on decomposition rates in mineral soils of subalpine grasslands. Plant Soil, 374, $579-591$

Hester A.J., Bergman M., Iason G.R., Moen J., 2006. Impacts of large herbivores on plant community structure and dynamics. In: Large herbivore ecology, ecosystem dynamics and conservation. Danell K., Duncan P., Bergström R., Pastor J. (Eds). Cambridge University Press, New York, USA, 97-141.

Hodge A., Robinson D., Fitter A., 2000. Are microorganisms more effective than plants at competing for nitrogen? Trends Plant Sci., 5, 304-308.

Hoekstra N.J., Bosker T., Lantinga E.A., 2002. Effects of cattle dung from farms with different feeding strategies on germination and initial root growth of cress (Lepidium sativum L.). Agricult. Ecosys. Environ., 93, 189-196.

Hofmann R.R., 1989. Evolutionary steps of ecophysiological adaptation and diversification of ruminants: a comparative view of their digestive system. Oecologia, 78, 443-457.

Hofmann R.R., Streich W.J., Fickel J., Hummel J., Clauss M., 2008. Convergent evolution in feeding types: salivary gland mass differences in wild ruminant species. J. Morphol., 269 240-257.

Holland J.N., Cheng W., Crossley D.A., 1996. Herbivore-induced changes in plant carbon allocation: assessment of below-ground $\mathrm{C}$ fluxes using carbon-14. Oecologia, 107, 87-94.

Huang Y., Wang L., Wang D., Zeng D.H., Liu C., 2016. How does the foraging behavior of large herbivores cause different associational plant defenses? Sci. Rep., 6, 20561.

Huntzinger M., Karban R., Young T.P., Palmer T.M., 2004. Relaxation of induced indirect defenses of acacias following exclusion of mammalian herbivores. Ecology, 85, 609-614.

Ibanez S., Bernard L., Coq S., Moretti M., Lavorel S., Gallet C., 2013. Herbivory differentially alters litter dynamics of two functionally contrasted grasses. Funct. Ecol., 27, 1064-1074.

Junker R.R., Blüthgen N., 2010. Floral scents repel facultative flower visitors, but attract obligate ones. Ann. Bot., 105, 777-782.

Kaplan I., Halitschke R., Kessler A., Sardanelli S., Denno R.F., 2008. Constitutive and induced defenses to herbivory in above-and belowground plant tissues. Ecology, 89, 392-406.

Kessler A., Baldwin I.T., 2002. Plant responses to insect herbivory: the emerging molecular analysis. Annu. Rev. Plant Biol., 53, 299-328.

Korpita T., Gómez S., Orians C.M., 2014. Cues from a specialist herbivore increase tolerance to defoliation in tomato. Funct. Ecol., 28, 395401 .

Kurokawa H., Nakashizuka T., 2008. Leaf herbivory and decomposability in a Malaysian tropical rain forest. Ecology, 89, 2645-2656.

Lefebvre T., 2016. Influence de 1'herbivorie sauvage et domestique en prairie subalpine : réponse métabolique des plantes et conséquences fonctionnelles sur la décomposition des litières. PhD thesis, Université Grenoble Alpes, France.
Lindgren Å., Klint J., Moen J., 2007. Defense mechanisms against grazing: a study of trypsin inhibitor responses to simulated grazing in the sedge Carex bigelowii. Oikos, 116, 1540-1546.

Liu J., Wang L., Wang D., Bonser S.P., Sun F., Zhou Y., Gao Y., Teng X., 2012. Plants can benefit from herbivory: stimulatory effects of sheep saliva on growth of Leymus chinensis. Plos One, 7, e29259.

Martin L.J., Agrawal A.A., Kraft C.E., 2014 Historically browsed jewelweed populations exhibit greater tolerance to deer herbivory than historically protected populations. J. Ecol., 103, 243-249.

Massey F.P., Ennos A.R., Hartley S.E., 2006 Silica in grasses as a defence against insect herbivores: contrasting effects on folivores and a phloem feeder. J. Anim. Ecol., 75, 595-603.

Milchunas D.G., Noy-Meir, I., 2002. Grazing refuges, external avoidance of herbivory and plant density. Oikos, 99, 113-130.

Milotic T., Hoffmann M., 2016. Cost or benefit for growth and flowering of seedlings and juvenile grassland plants in a dung environment. Plant. Ecol., 217, 1025-1042.

Mithöfer A., Boland W., 2012. Plant defense against herbivores: chemical aspects. Annu. Rev. Plant Biol., 63, 431-450.

Moore K.J., Jung H.J.G., 2001. Lignin and fiber digestion. J. Range Manage., 54, 420-430.

Mueller-Harvey I., 2006. Unravelling the conundrum of tannins in animal nutrition and health. J. Sci. Food Agric., 86, 2010-2037.

Newingham B.A., Callaway R.M., BassiriRad H., 2007. Allocating nitrogen away from a herbivore: a novel compensatory response to root herbivory. Oecologia, 153, 913-920.

Nolte S., Esselink P., Bakker J.P., Smit C., 2015. Effects of livestock species and stocking density on accretion rates in grazed salt marshes. Estuar. Coast. Shelf. Sci., 152, 109-115

Olofsson J., Oksanen L., 2002. Role of litter decomposition for the increased primary production in areas heavily grazed by reindeer: a litterbag experiment. Oikos, 96, 507-515.

Parachnowitsch A.L., Caruso C.M., Campbell S.A., Kessler A., 2012. Lobelia siphilitica plants that escape herbivory in time also have reduced latex production. Plos One, 7, e37745.

Pastor J., Dewey B., Naiman R.J., McInnes P.F., Cohen Y., 1993. Moose browsing and soil fertility in the boreal forests of isle royale national park. Ecology, 74, 467-480.

Pellissier F., 2013. Early physiological responses of Abies alba and Rubus fruticosus to ungulate herbivory. Plant Ecol., 214, 127-138.

Pfister J.A., Panter K.E., Gardner D.R., Stegelmeier B.L., Ralphs M.H., Molyneux R.J., Lee S.T., 2001. Alkaloids as anti-quality factors in plants on western U.S. rangelands. J. Range Manage., 54, 447-461.

Provenza F.D., Villalba J.J., Haskell J., MacAdam J.W., Griggs T.C., Wiedmeier R.D. 2007. The value to herbivores of plant physical and chemical diversity in time and space. Crop Sci., 47, 382-398.

Redondo-Gómez S., Mancilla-Leytón J.M., Mateos-Naranjo E., Cambrollé J., Martín-
Vicente A., 2010. Differential photosynthetic performance of three Mediterranean shrubs under grazing by domestic goats. Photosynthetica, $48,348-354$

Risch A.C., Haynes A.G., Busse M.D., Filli F., Schütz M., 2013. The response of soil $\mathrm{CO}_{2}$ fluxes to progressively excluding vertebrate and invertebrate herbivores depends on ecosystem type. Ecosystems, 16, 1192-1202.

Sankaran M., Augustine D.J., 2004. Large herbivores suppress decomposer abundance in a semiarid grazing ecosystem. Ecology, 85, 10521061

Schimel J.P., Cates R.G., Ruess R., 1998. The role of balsam poplar secondary chemicals in controlling soil nutrient dynamics through succession in the Alaskan taiga. Biogeochemistry, $42,221-234$

Schrama M., Heijning P., Bakker J.P., Wijnen H.J., Berg M.P., Olff H., 2013. Herbivore trampling as an alternative pathway for explaining differences in nitrogen mineralization in moist grasslands. Oecologia, 172, 231-243.

Semmartin M., Aguiar M.R., Distel R.A., Moretto A.S., Ghersa C.M., 2004. Litter quality and nutrient cycling affected by grazing-induced species replacements along a precipitation gradient. Oikos, 107, 148-160.

Shimada T., 2006. Salivary proteins as a defense against dietary tannins. J. Chem. Ecol., 32 1149-1163.

Ssemakul J., 1983. A comparative study of hoof pressures of wild and domestic ungulates. Afr. J. Ecol., 21, 325-328.

Stark S., Wardle D.A., Ohtonen R., Helle T., Yeates G.W., 2000. The effect of reindeer grazing on decomposition, mineralization and soil biota in a dry oligotrophic scots pine forest. Oikos, 90, 301-310.

Strauss S.Y., Agrawal A.A., 1999. The ecology and evolution of plant tolerance to herbivory. Trends Ecol. Evol., 14, 179-185.

Sui Y., He W., Pan X., Dong M., 2011. Partial mechanical stimulation facilitates the growth of the rhizomatous plant leymus secalinus: modulation by clonal integration. Ann. Bot., 107, 693-697.

Thomson V.P., Cunningham S.A., Ball M.C., Nicotra A.B., 2003. Compensation for herbivory by Cucumis sativus through increased photosynthetic capacity and efficiency. Oecologia, $134,167-175$

Turlings T.C.J., Tumlison J.H., Lewis W.J., 1990. Exploitation of herbivore-induced plant odors by host-seeking parasitic wasps. Science, 250, 1251-1253.

Wardle D.A., Bonner K.I., Barker G.M., 2002. Linkages between plant litter decomposition, litter quality, and vegetation responses to herbivores. Funct. Ecol., 16, 585-595.

Wardle D.A., Bardgett R.D., Klironomos J.N., Setälä H., van der Putten W.H., Wall D.H., 2004. Ecological linkages between aboveground and belowground biota. Science, 304, 1629-1633.

Xu L., Freitas S.M.A., Yu F.-H., Dong M., Anten N.P.R., Werger M.J.A., 2013. Effects of trampling on morphological and mechanical traits of dryland shrub species do not depend on water availability. Plos One, 8, e53021. 


\title{
Résumé
}

En tant que consommateurs primaires, les herbivores jouent un rôle essentiel dans la dynamique de la végétation. Leur influence peut se manifester de l'échelle de la plante, via la modification de son métabolisme, jusqu'à l'échelle de la communauté végétale, par un changement de sa composition floristique ou de l'abondance des espèces qui la composent. Quelle que soit l'échelle considérée, les effets sur la végétation peuvent se répercuter sur la qualité des litières et ainsi impacter le processus de décomposition. Il en résulte souvent une modification des cycles biogéochimiques et plus largement du fonctionnement de l'écosystème. Cet article se propose de faire l'état de la littérature concernant les interactions entre plantes et grands herbivores, en développant plus particulièrement les moyens par lesquels les herbivores affectent les plantes, les stratégies mises en place par ces dernières pour se défendre, ainsi que les processus par lesquels les herbivores peuvent influencer le fonctionnement de l'écosystème via la décomposition des litières.

\begin{abstract}
Impacts of large herbivores on plants: consequences for litter decomposition

As primary consumers, herbivores play an important role in vegetation dynamics. Their effects run from the plant level by influencing plant metabolism, to the community level by changing the floristic composition and the abundance of plant species. These effects on vegetation can impact litter quality and can thus alter the decomposition process. This can in turn impact soil biogeochemical cycles and modify ecosystem functioning. Here, we review the literature on plant and large herbivore interactions, focusing on the means by which herbivores affect plants, the plant strategies to cope with herbivory, and the mechanisms by which herbivores can affect ecosystem functioning via the alteration of litter decomposition.
\end{abstract}

LEFEBVRE T., GALLET C., 2017. Impacts des grands herbivores sur la végétation des prairies et conséquences sur la décomposition de la litière. INRA Prod. Anim., 30, 455-464. 